\title{
Cloning, Expression and Characterization of Mitochondrial Manganese Superoxide Dismutase from the Whitefly, Bemisia tabaci
}

\author{
Xian-Long Gao ${ }^{1}$, Jun-Min Li ${ }^{2}$, Yong-Liang Wang ${ }^{1}$, Min Jiu ${ }^{3}$, Gen-Hong Yan ${ }^{1}$, \\ Shu-Sheng Liu ${ }^{1}$ and Xiao-Wei Wang ${ }^{1, *}$
}

1 Ministry of Agriculture Key Laboratory of Agricultural Entomology, Institute of Insect Sciences, Zhejiang University, Hangzhou 310058, China; E-Mails: gx1060308024@163.com (X.-L.G.); wangyongliang2006a@126.com (Y.-L.W.); ghyan@zju.edu.cn (G.-H.Y.); shshliu@zju.edu.cn (S.-S.L.)

2 Institute of Virology and Biotechnology, Zhejiang Academy of Agricultural Sciences, Hangzhou 310021, China; E-Mail: zjlijunmin@gmail.com

3 College of Food and Bioengineering, Henan University of Science and Technology, Luoyang 471023, China; E-Mail: jiumin0912@163.com

* Author to whom correspondence should be addressed; E-Mail: xwwang@zju.edu.cn; Tel.: +86-571-8898-2435; Fax: +86-571-8898-2355.

Received: 7 October 2012; in revised form: 21 November 2012 / Accepted: 24 December 2012 / Published: 7 January 2013

Abstract: A mitochondrial manganese superoxide dismutase from an invasive species of the whitefly Bemisia tabaci complex (Bt-mMnSOD) was cloned and analyzed. The full length cDNA of Bt-mMnSOD is 1210 bp with a 675 bp open reading frame, corresponding to 224 amino acids, which include 25 residues of the mitochondrial targeting sequence. Compared with various vertebrate and invertebrate animals, the MnSOD signature (DVWEHAYY) and four conserved amino acids for manganese binding (H54, H102, D186 and H190) were observed in Bt-mMnSOD. Recombinant Bt-mMnSOD was overexpressed in Escherichia coli, and the enzymatic activity of purified mMnSOD was assayed under various temperatures. Quantitative real-time PCR analysis with whiteflies of different development stages showed that the mRNA levels of Bt-mMnSOD were significantly higher in the 4th instar than in other stages. In addition, the in vivo activities of MnSOD in the whitefly were measured under various conditions, including exposure to low $\left(4{ }^{\circ} \mathrm{C}\right)$ and high $\left(40^{\circ} \mathrm{C}\right)$ temperatures, transfer from a favorable to an unfavorable host plant (from cotton to tobacco) and treatment with pesticides. Our results indicate that the 
whitefly MnSOD plays an important role in cellular stress responses and anti-oxidative processes and that it might contribute to the successful worldwide distribution of the invasive whitefly.

Keywords: mitochondrial manganese superoxide dismutase; oxidative stress; whitefly; Bemisia tabaci

\section{Introduction}

The whitefly Bemisia tabaci (Gennadius) (Hemiptera: Aleyrodidae) is a species complex with global distribution, and some members of the complex cause severe damage to the production of vegetables, fibers and ornamental crops [1-4]. A major recent event associated with $B$. tabaci has been the global invasion of Middle East-Asia Minor 1 specie (MEAM1, formerly known as the "B biotype") that has been ranked among the top 100 invasive species [5-7]. MEAM1, one of the 24 putative species delineated by Dinsdale et al. [8], invaded China in the mid-1990s [9-11] and gradually displaced indigenous $B$. tabaci species during its invasion in many regions [7,12]. Previous studies indicated that the invasive ability of MEAM1 may be closely related to its high capability of surviving under different stress conditions [1,13]. And recently, reports showed that the activity of superoxide dismutase might be associated with the ability of invasive whitefly to survive under various stress conditions, such as extreme temperatures and unfavorable host plants [14,15].

Superoxide dismutases (SODs) are a family of metalloenzymes that catalyze the dismutation of reactive oxygen species (ROS) [16], which can be toxic to nucleic acids, proteins and membrane lipids $[17,18]$. Three major types of SODs have been characterized based on their metal content: copper/zinc SOD (CuZnSOD), manganese SOD (MnSOD) and iron SOD (FeSOD) [19,20]. They are widely distributed in bacteria, fungi, plants and animals [21].

MnSOD is primarily found in prokaryotes and eukaryotic mitochondria [22]. Two types of MnSOD are known in eukaryotes: mitochondrial MnSOD (mMnSOD), with a mitochondrial transit peptide for translocation, and cytosolic MnSOD (cMnSOD) without the peptide [23]. In eukaryotic cells, $\mathrm{mMnSOD}$ is synthesized as a precursor protein in the cytosol and then imported into the mitochondrial matrix [21]. The mMnSOD is located in mitochondria and plays a vital role in defense against superoxide radicals generated as byproducts of oxidative phosphorylation [24]. cMnSOD has been identified and cloned in various crustacean species. The characterization and its role in immunomodulation have also been reported [19,25,26]. Preliminary studies showed that the overexpression of MnSOD can extend the life span of adult Drosophila melanogaster [27] and reduce oxidative stress in cells [28,29]. Recently, it was found that MnSOD may be involved in immune responses to stimulators, such as heat, coldness, starvation and heavy metals [30,31]. However, until now, information about the function of MnSOD in B. tabaci was still lacking.

Previously, we found that $\mathrm{CuZnSOD}$ plays a major role in protecting the whitefly $B$. tabaci against various stress conditions [15]. To gain further insights into the characteristics of MnSOD and its functional roles in the defense system, we cloned the mMnSOD gene of B. tabaci and purified the recombinant protein expressed in Escherichia coli. We then performed biochemical assays to elucidate 
the activity of the recombinant protein. Next, we examined the mRNA levels of Bt-mMnSOD in different developmental stages of the whitefly. Finally, we investigated the in vivo activities of MnSOD in the whitefly under various stress conditions including heat, coldness, shift of host plants and pesticide treatments.

Figure 1. Nucleotide and deduced amino acid sequences of mitochondrial manganese superoxide dismutase from Bemisia tabaci (Bt-mMnSOD). The start codon (ATG), stop codon (TAG) and putative polyadenylation signal (AATAAA) are boxed. Putative mitochondrial targeting sequence is underlined. The manganese superoxide dismutase (MnSOD) signature motif (DVWEHAYY) is shown in bold red letters. Four putative manganese binding sites (H54, H102, D186 and H190) are circled.

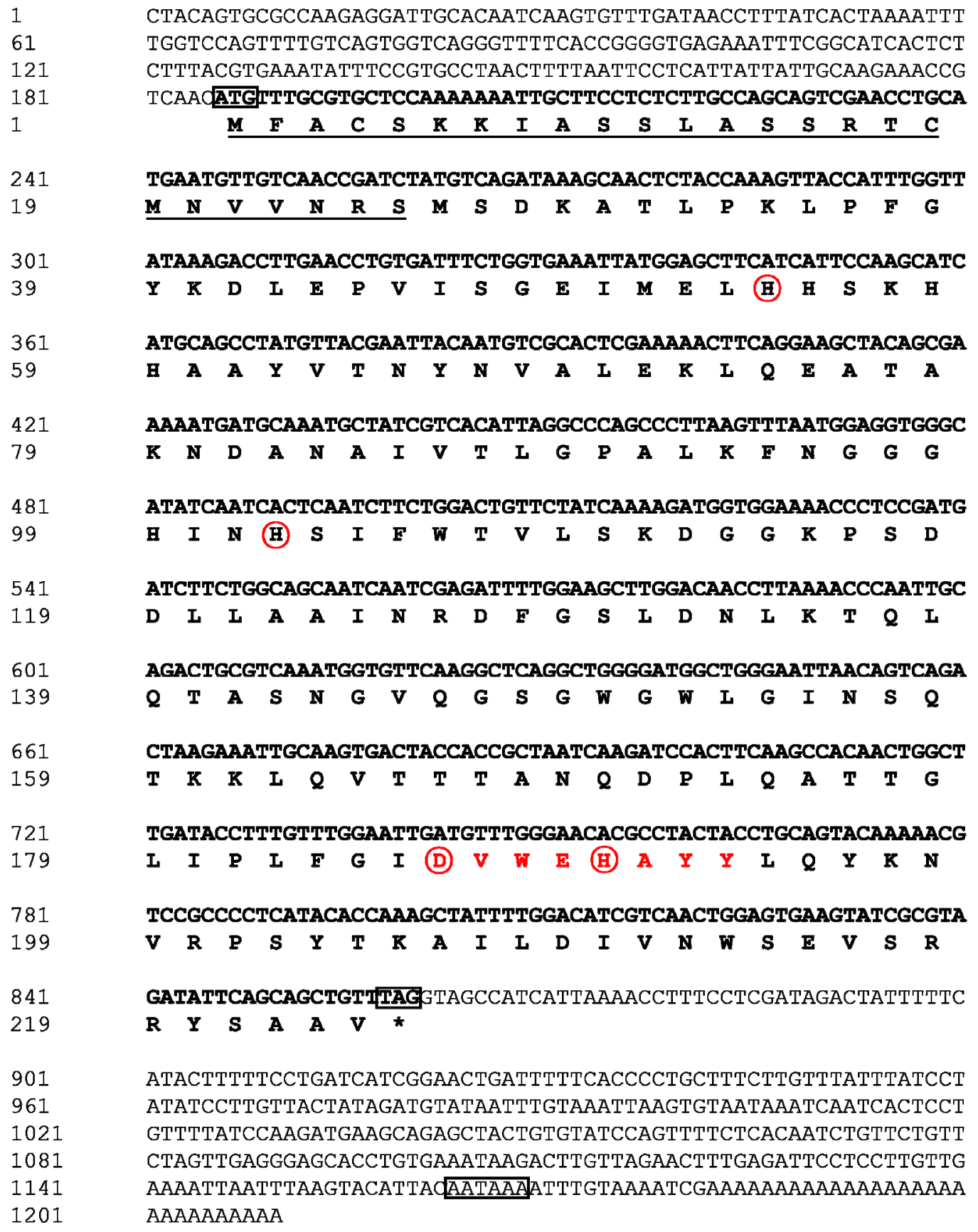

ATACTTTTTCCTGATCATCGGAACTGATTTTTCACCCCTGCTTTCTTGTTTATTTATCCT ATATCCTTGTTACTATAGATGTATAATTTGTAAATTAAGTGTAATAAATCAATCACTCCT GTTTTATCCAAGATGAAGCAGAGCTACTGTGTATCCAGTTTTCTCACAATCTGTTCTGTT CTAGTTGAGGGAGCACCTGTGAAATAAGACTTGTTAGAACTTTGAGATTCCTCCTTGTTG AAAATTAATTTAAGTACATTA AATAAAATTGTAAAATCGAAAAAAAAAAAAAAAAAAA AAAAAAAAAA 


\section{Results}

\subsection{Analysis of the Bt-mMnSOD Sequence and the Predicted Protein}

The full-length mMnSOD cDNA of B. tabaci (GenBank accession number JQ867105) consists of $1210 \mathrm{bp}$, with a $675 \mathrm{bp}$ open reading frame (ORF), which encodes 224 amino acids. The full-length nucleotide sequences and the deduced amino acid sequences are shown in Figure 1. The Bt-mMnSOD cDNA sequence contains a 5' untranslated region (UTR) of 185 nucleotides, a long 3' UTR of 350 nucleotides consisting of a stop codon (TAG), a putative polyadenylation consensus signal (AATAAA) and a poly (A) tail. The predicted molecular mass is $24.5 \mathrm{kDa}$ and the estimated isoelectric point of this protein is 8.97. SignalP program analysis showed no putative signal peptide in Bt-mMnSOD. However, TargetP and MITOPROT revealed that it contains a putative mitochondrial targeting sequence of 25 amino acids and is located inside the mitochondria.

Multiple sequences alignment of Bt-mMnSOD with other MnSODs of vertebrate and invertebrate animals indicated high conservation of four putative manganese binding sites (H54, H102, D186 and H190) and a MnSOD signature from 186 to 193 (DVWEHAYY) (Figure 2). The major differences between the mitochondrial and cytosolic MnSODs (cMnSODs) are located at the $N$-terminal region (Figure 2). cMnSODs have a conserved $N$-terminal extension, which is not present in mMnSODs, but lack a mitochondrial targeting sequence. MnSOD sequences of whitefly and other species were obtained from GenBank (Table 1).

Table 1. Sequences of MnSOD used in this study.

\begin{tabular}{|c|c|c|c|}
\hline Species & Protein & Abbreviation & GenBank no. \\
\hline Homo sapiens & mitochondrial MnSOD & H. sapiens-mMnSOD & P04179.2 \\
\hline Cavia porcellus & mitochondrial MnSOD & C. porcellus-mMnSOD & XM_003466367.1 \\
\hline Pongo abelii & mitochondrial MnSOD & P. abelii-mMnSOD & NM_001133563.1 \\
\hline Taeniopygia guttata & mitochondrial MnSOD & T. guttata-mMnSOD & DQ214967.1 \\
\hline Gallus gallus & mitochondrial MnSOD & G. gallus-mMnSOD & NM_204211.1 \\
\hline Melopsittacus undulatus & mitochondrial MnSOD & M. undulatus-mMnSOD & AY241394.1 \\
\hline Danio rerio & mitochondrial MnSOD & D. rerio-mMnSOD & NP_956270.1 \\
\hline Hemibarbus mylodon & mitochondrial MnSOD & H. mylodon-mMnSOD & ACR23311.1 \\
\hline Hypophthalmichthys nobilis & mitochondrial MnSOD & H. nobilis-mMnSOD & ADM26563.1 \\
\hline Bemisia tabaci & mitochondrial MnSOD & B. tabaci-mMnSOD & JQ867105 \\
\hline Acyrthosiphon pisum & mitochondrial MnSOD & A. pisum-mMnSOD & NM_001246048.1 \\
\hline Caenorhabditis elegans & mitochondrial MnSOD & C. elegans-mMnSOD & D12984.1 \\
\hline Ancylostoma duodenale & mitochondrial MnSOD & A. duodenale-mMnSOD & FJ465146.1 \\
\hline Haliotis discus discus & mitochondrial MnSOD & H. discus-mMnSOD & DQ821491.1 \\
\hline Mizuhopecten yessoensis & mitochondrial MnSOD & M. yessoensis-mMnSOD & AB222783.1 \\
\hline \multirow[t]{2}{*}{ Callinectes sapidus } & mitochondrial MnSOD & C. sapidus-mMnSOD & AF264029.1 \\
\hline & cytosolic MnSOD & C. sapidus-cMnSOD & AAF74771.1 \\
\hline \multirow[t]{2}{*}{ Scylla paramamosain } & mitochondrial MnSOD & S. paramamosain-mMnSOD & FJ605170.2 \\
\hline & cytosolic MnSOD & S. paramamosain-cMnSOD & ADA63848.1 \\
\hline Portunus trituberculatus & cytosolic MnSOD & P. trituberculatus-cMnSOD & FJ031018.1 \\
\hline Fenneropenaeus chinensis & cytosolic MnSOD & F. chinensis-cMnSOD & ACS49842.1 \\
\hline Litopenaeus vannamei & cytosolic MnSOD & L. vannamei-cMnSOD & DQ005531.1 \\
\hline
\end{tabular}


Figure 2. Multiple alignments of the deduced amino acid sequence of the Bt-mMnSOD with the mitochondrial MnSOD (mMnSODs) and cytosolic MnSOD (cMnSODs) of other species. The manganese superoxide dismutase signature DVWEHAYY is boxed in red (labeled Signature), and the amino acids required for binding $\mathrm{Mn}$ are indicated with arrows.

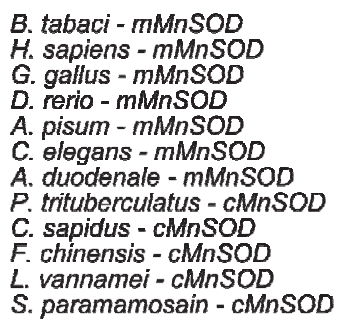

B. tabaci-mMnSOD

H. sapiens - $m M n S O D$

G. gallus - $m M n S O D$

D. rerio - $m M n S O D$

A. pisum - $m M n S O D$

C. elegans - $m M n S O D$

A. duodenale $-m M n S O D$

$P$. trituberculatus - cMnSOD

C. sapidus - cMnSOD

$F$. chinensis - cMnSOD

L. vannamei - $c M n S O D$

S. paramamosain - cMnSOD

B. tabaci-mMnSOD

H. sapiens - $m M n S O D$

$G$. gallus - $m M n S O D$

D. rerio - $m M n S O D$

A. pisum - $m M n S O D$

C. elegans - $m M n S O D$

A. duodenale - $m M n S O D$

$P$. trituberculatus - CMnSOD

C. sapidus - cMnSOD

$F$. chinensis - cMnSOD

L. vannamei - cMnSOD

S. paramamosain - cMnSOD
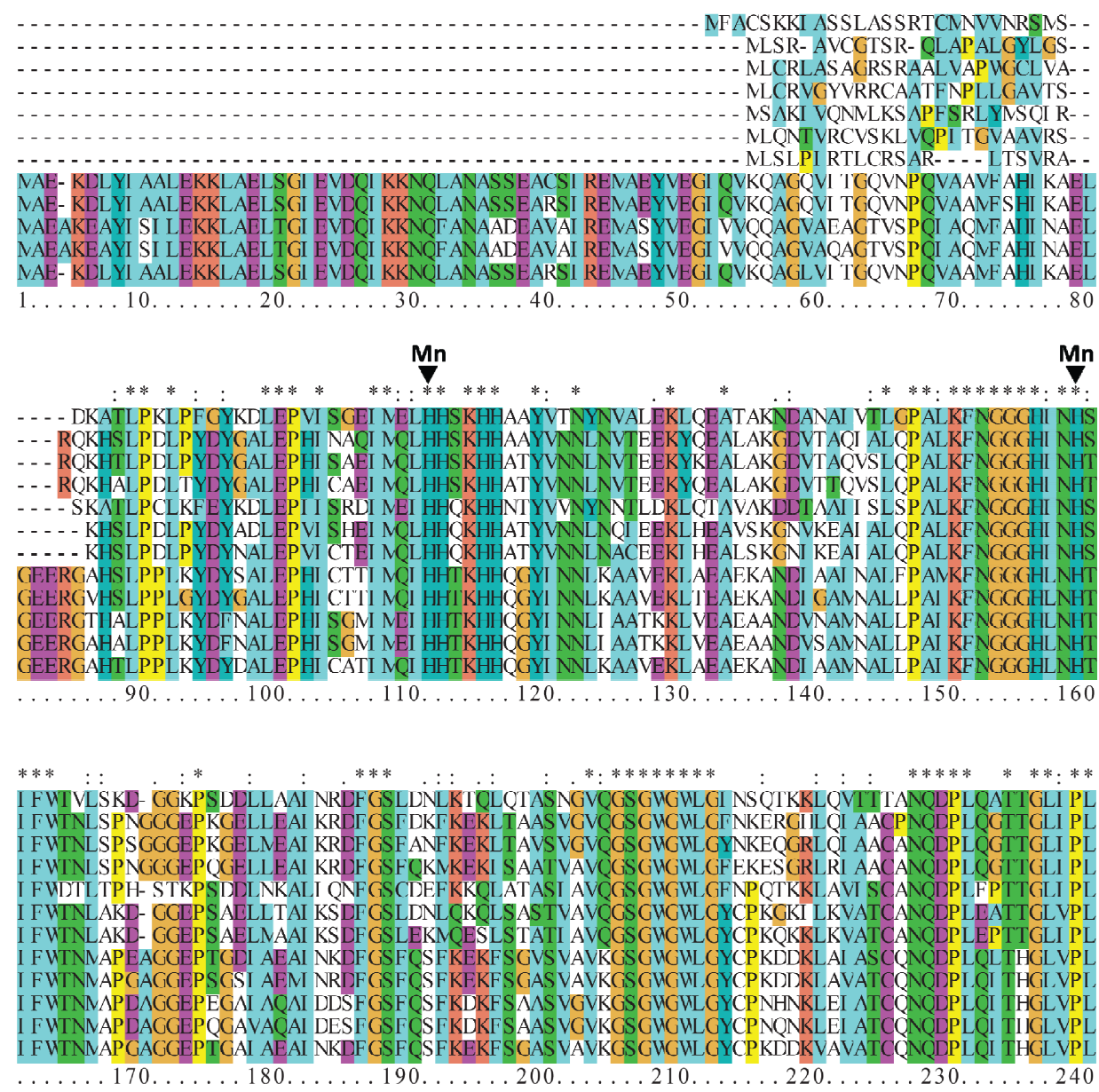

\section{Signature}

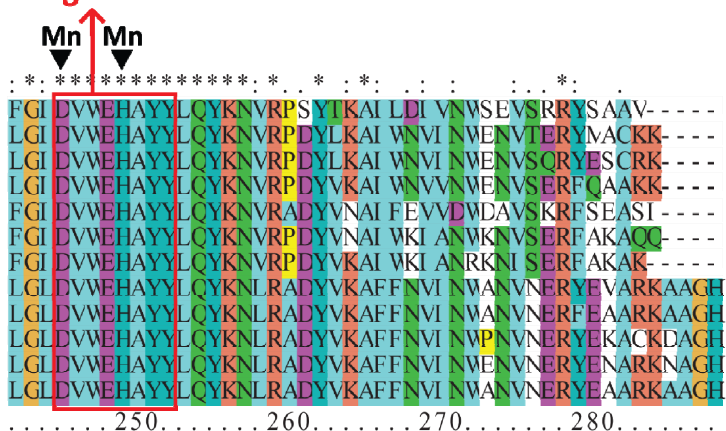

A phylogenetic tree of the amino acid sequences of selected MnSODs was constructed by the neighbor-joining distance method using MEGA version 4 (Figure 3). The phylogenetic analysis indicated that MnSODs can be classified into two groups. All the mMnSODs were clustered together as one subgroup and all cMnSODs as another group (Figure 3). Bt-mMnSOD was in the same group of other mMnSODs, suggesting that it is an mMnSOD. 
Figure 3. Phylogenetic relationships between mMnSODs and cMnSODs from 22 different species. A phylogenetic analysis was conducted based on a multiple alignment of amino acid sequences retrieved from the GenBank database. Numbers at the nodes are bootstrap values (1000 replications). The scale bar is 0.1 .

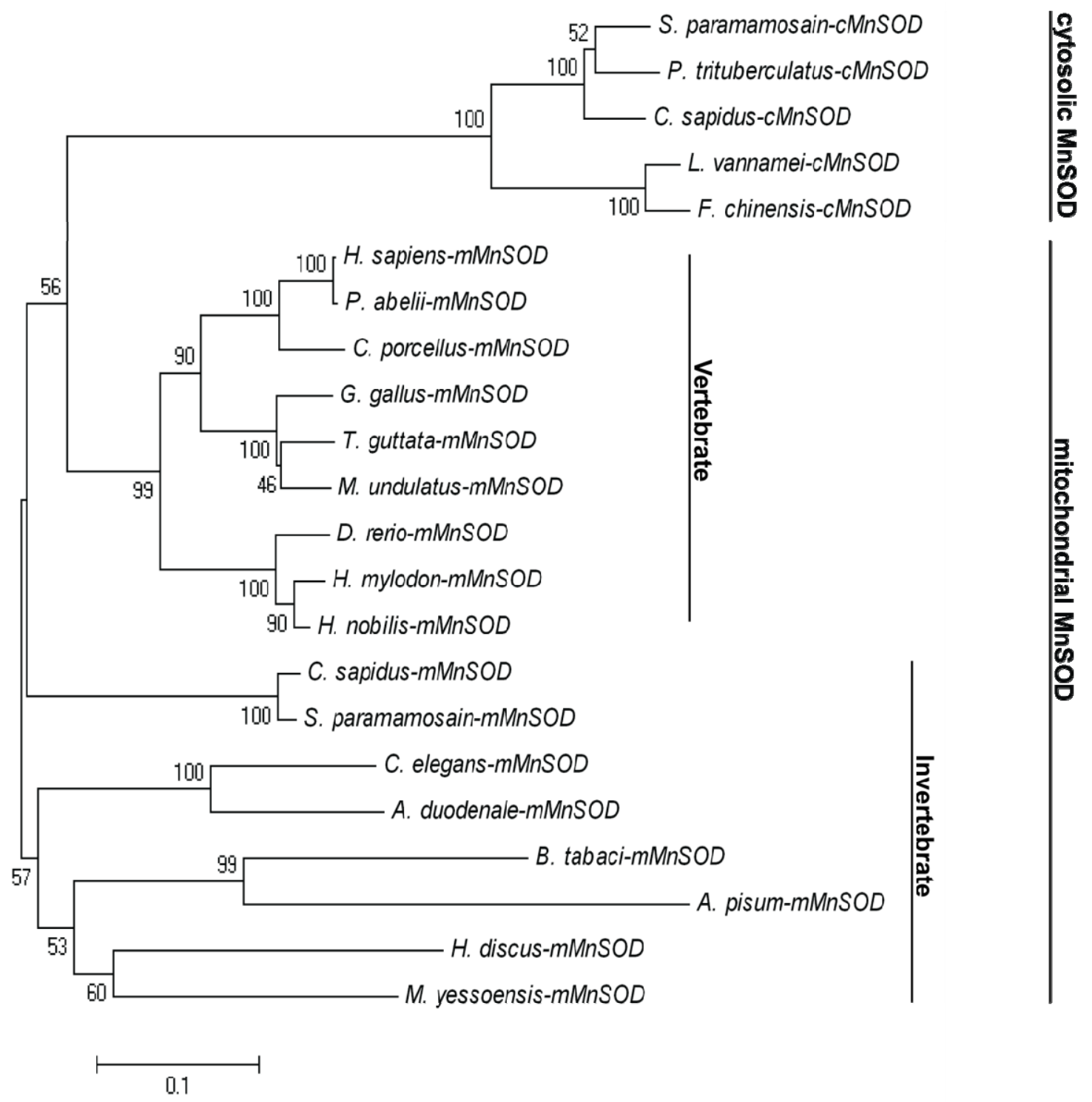

\subsection{Overexpression and Purification of Bt-mMnSOD}

The expression of Bt-mMnSOD protein was considerably increased after induction with $0.2 \mathrm{mM}$ IPTG at $18{ }^{\circ} \mathrm{C}$ for $18 \mathrm{~h}$ (Figure 4, lanes 1, 2). Moreover, the proteins were expressed both in the inclusion bodies and as soluble protein (Figure 4, lanes 3, 4). The soluble Bt-mMnSOD protein was then purified with GST purification system and resolved by SDS-PAGE, with the result of a $50.5 \mathrm{kDa}$ single band (Figure 4, lane 5). The molecular mass of the purified product was consistent with the predicted molecular weight of the recombinant protein, which consisted of Bt-mMnSOD (24.5 $\mathrm{kDa})$ and GST tag $(26 \mathrm{kDa})$. 
Figure 4. Expression and purification of superoxide dismutase (SOD) recombinant protein. Lane $\mathrm{M}$, molecular weight marker; lane 1, un-induced total protein; lane 2, total protein induced with $0.2 \mathrm{mM}$ isopropyl $\beta$-D-1-thiogalactopyranoside (IPTG) for $18 \mathrm{~h}$ at $18{ }^{\circ} \mathrm{C}$; lane 3, insoluble proteins; lane 4, soluble proteins; lane 5, purified recombinant protein of GST-Bt-mMnSOD.

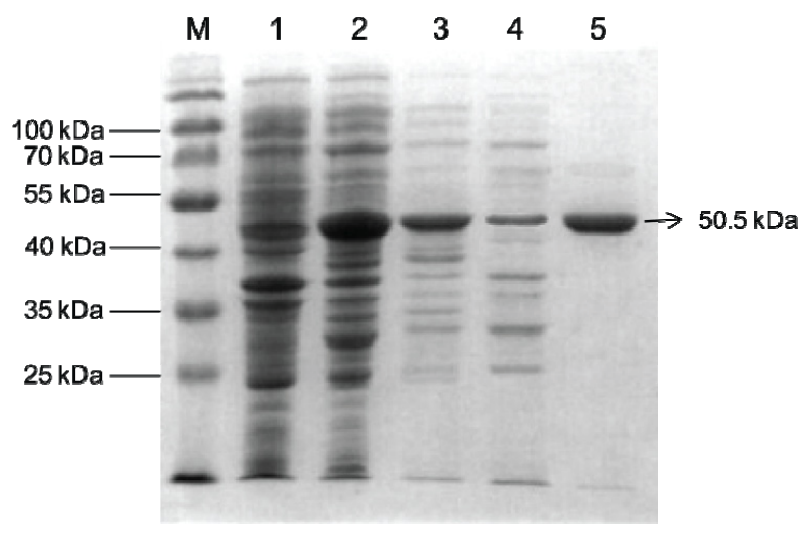

\subsection{Thermostability of Purified Bt-mMnSOD}

Thermostability of purified Bt-mMnSOD was studied by incubating the enzyme at 37, 45, 55, 65, 75 and $85{ }^{\circ} \mathrm{C}$ for various intervals. The residual activities of Bt-mMnSOD were measured (Figure 5). The enzymatic activity of Bt-mMnSOD was stable after incubation at $37{ }^{\circ} \mathrm{C}$ and $45{ }^{\circ} \mathrm{C}$ for $30 \mathrm{~min}$. At the temperature of $55^{\circ} \mathrm{C}$, the Bt-mMnSOD activity decreased significantly after $20 \mathrm{~min}(p<0.01)$, but still retained up to $70 \%$ after 60 min incubation. The activity reduced by $50 \%$ when incubated for $10 \mathrm{~min}$ at $65^{\circ} \mathrm{C}$ and decreased rapidly in the initial $10 \mathrm{~min}(p<0.01)$ at 75 and $85{ }^{\circ} \mathrm{C}$. The enzyme was completely inactivated when incubated for $50 \mathrm{~min}$ at $85^{\circ} \mathrm{C}$ and $60 \mathrm{~min}$ at $75^{\circ} \mathrm{C}$, respectively.

Figure 5. Effects of temperature on the purified recombinant Bt-mMnSOD activity. Each bar represents the mean $\pm \mathrm{SD}(n=3)$. For each temperature, significant differences are indicated by different letters at $p<0.01$ (least significant difference (LSD) test).

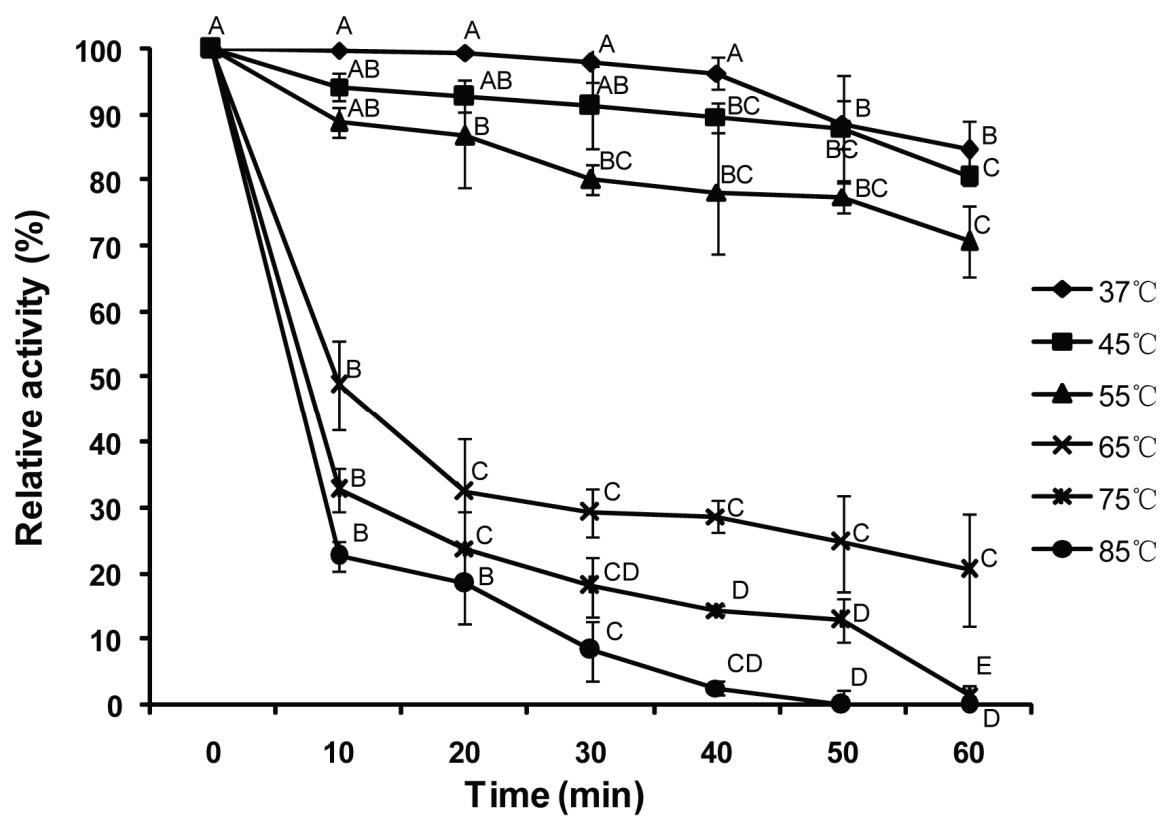




\subsection{Expression of Bt-mMnSOD during Development}

To better understand the physiological roles of Bt-mMnSOD, its expression was detected by qPCR in different developmental stages. The mRNA levels of Bt-mMnSOD were significantly higher in the 4th instar than in egg and nymph or adult (Figure 6). The result of qPCR suggests that Bt-mMnSOD might play important roles in the development of B. tabaci, especially in the 4th instar.

Figure 6. Quantification of mitochondrial manganese zinc superoxide dismutase from Bemisia (Bt-mMnZnSOD) gene expression in different developmental stages. Each symbol and vertical bar represent the mean $\pm \operatorname{SD}(n=3)$.

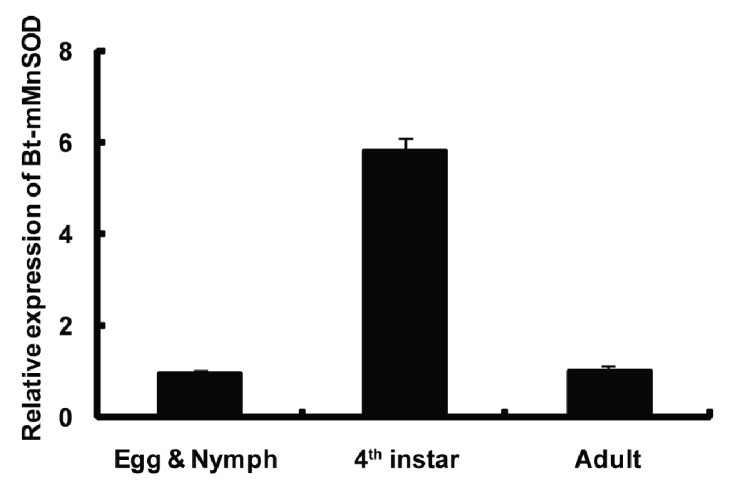

\subsection{Effect of Thermal Stresses, Host Shift and Pesticide Treatment on MnSOD Activity}

To evaluate the in vivo activity of the MnSOD in $B$. tabaci stimulated by external temperature stresses, B. tabaci adults were exposed to low $\left(4{ }^{\circ} \mathrm{C}\right)$, medium $\left(26^{\circ} \mathrm{C}\right)$ and high $\left(40{ }^{\circ} \mathrm{C}\right)$ temperatures for 0 (control), 30, 60 and $120 \mathrm{~min}$, respectively (Figure 7). The activity of MnSOD significantly increased after incubation at low $\left(4{ }^{\circ} \mathrm{C}\right)$ and high $\left(40{ }^{\circ} \mathrm{C}\right)$ temperatures for 60 and 120 min, compared with control $(p<0.01)$. However, no significant differences were detected when the $B$. tabaci adults were exposed at $26^{\circ} \mathrm{C}$ from 0 to $120 \mathrm{~min}$ or incubated at $4{ }^{\circ} \mathrm{C}$ and $40{ }^{\circ} \mathrm{C}$ in the initial $30 \mathrm{~min}$.

Figure 7. Effect of thermal stresses to MnSOD activity of whitefly in vivo. Each bar represents the mean $\pm \mathrm{SD}$ of MnSOD activity $(n=3)$. Compared with control ( $0 \mathrm{~min})$, significant differences of each temperature at different sampling times are indicated by two asterisks at $p<0.01$ (LSD test).

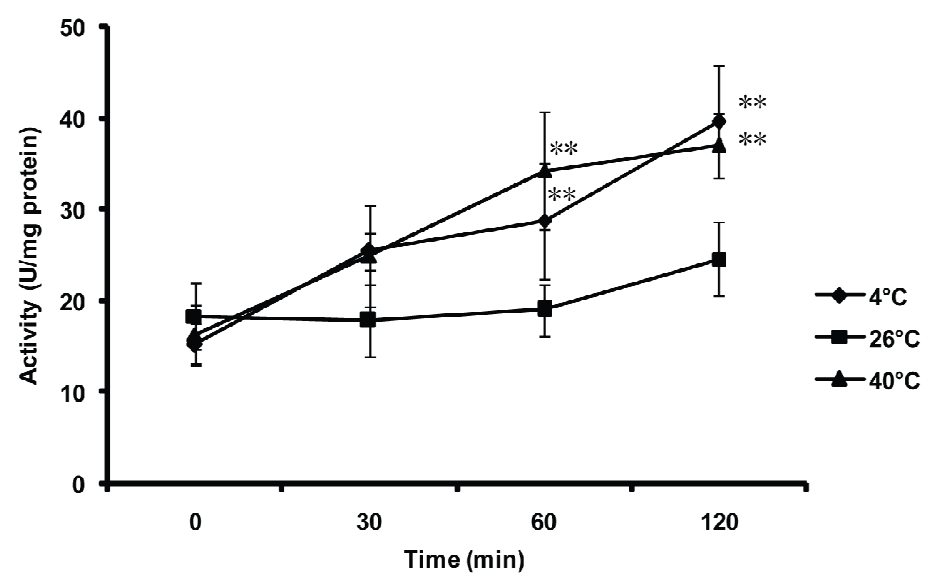


To characterize the effect of host shift on the in vivo activity of the MnSOD in B. tabaci, whitefly adults were transferred from cotton (a favorable host plant) to tobacco (an unfavorable host plant) for $0,1,3$ and 5 days. When whiteflies were transferred from cotton to tobacco for 5 days, the activity of MnSOD significantly increased compared with 0 days $(p<0.01)$, whereas in the control (transfer from cotton to cotton), the level of activity remained unchanged (Figure 8 ).

Figure 8. Effect of host shift on MnSOD activity of whitefly in vivo. Each symbol and bar represents the mean $\pm \mathrm{SD}$ of MnSOD activity $(n=3)$. Significant differences between various sampling times of each host plant compared with 0 days are indicated by two asterisks at $p<0.01$ (LSD test).

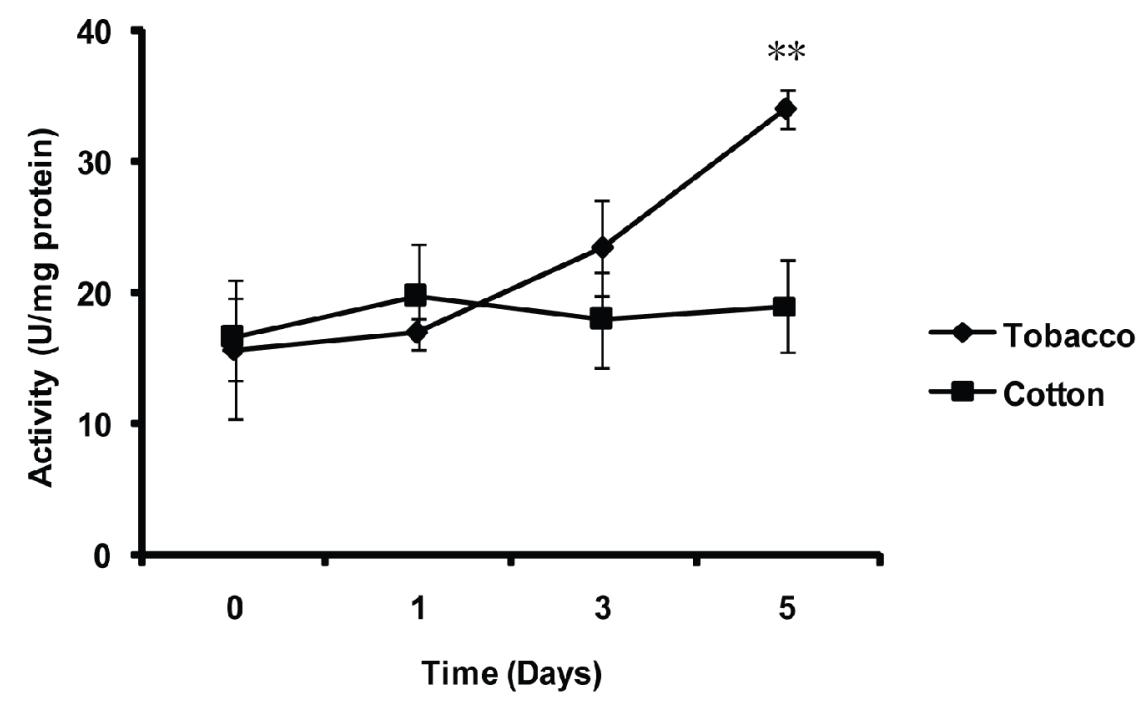

Furthermore, the activities of MnSOD were measured after the whitefly feeding on the imidacloprid-treated cotton plants for various durations (Figure 9). No significant differences were observed in the initial $12 \mathrm{~h}$. However, after $24 \mathrm{~h}$ feeding on imidacloprid-treated cotton plants, a significant increase of MnSOD activity was detected compared with $0 \mathrm{~h}(p<0.01)$.

Figure 9. Effect of pesticide on MnSOD activity of whitefly in vivo. Each bar represents the mean $\pm \mathrm{SD}(n=3)$. Significant differences between various times compared with $0 \mathrm{~h}$ are indicated by two asterisks at $p<0.01$ (LSD test).

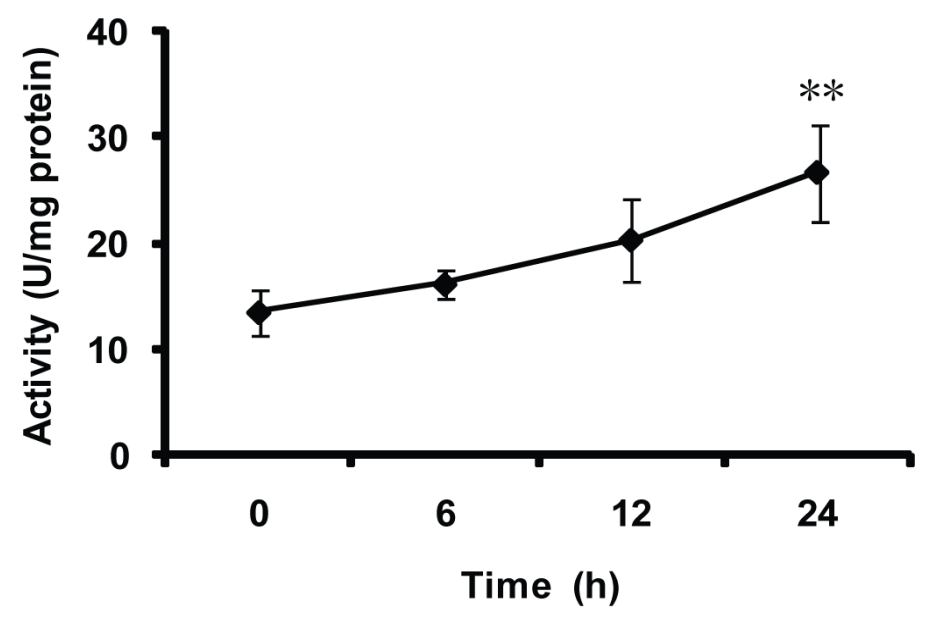




\section{Discussion}

Reactive oxygen species are formed in the body as natural products of oxidative metabolism. ROS cause oxidative stress and can be toxic to many cell components [17]. The mitochondrial matrix is the major site for the production of superoxide radicals. MnSOD is known to be a key antioxidant enzyme in the mitochondrial matrix, which can catalyze the dismutation of the superoxide radicals into hydrogen peroxide [32,33].

MnSOD genes have been cloned from various organisms, but few data are available regarding the mMnSOD from B. tabaci. In the present study, a mMnSOD cDNA was identified from B. tabaci. Multiple sequences alignment of mMnSODs and cMnSODs from various species shows that the four metal-binding residues and MnSOD signature are highly conserved, indicating that these sites are essential to the structure and function of MnSODs. The identification of the signature sequence and conserved metal-binding residues suggested that the Bt-mMnSOD possesses the essential properties of MnSOD family. MITOPROT analysis revealed it contains a putative mitochondrial targeting sequence of 25 amino acids, which can translocate the mMnSOD into the mitochondrial matrix $[21,23]$. For phylogeny analysis, MnSODs from different species were found to cluster into two groups with robust separate branches of cMnSOD and mMnSOD. Bt-mMnSOD clustered with the mMnSOD group, suggesting that it is a mitochondrial MnSOD.

Temperature stress was reported as one of the key mediators of ROS generation [34,35]. Previous studies have shown that temperature stress can cause MnSOD induction in several species: Chinese hamster lung fibroblast cell [36], Hydra vulgaris [37] and Hyphantria cunea [30]. An earlier study indicated that the activity of CuZnSOD in $B$. tabaci was noticeably enhanced under cold and heat stress [15]. In this study, we found that the MnSOD activity of B. tabaci was also increased with the aforementioned conditions. These results indicated that both MnSOD and CuZnSOD may function synergistically as scavengers to remove the intracellular superoxide anions generated under high or low temperatures, which might be related to the MEAM1 invasive ability.

Species of phytophagous insects differ in preference, ingestion speed, digestion efficiency and detoxification of the ingredients ingested towards different host plants, leading to the variability in their ability of host utilization [38,39]. Therefore, host shift might affect the individual performance of insects. In our study, we found that the in vivo activity of MnSOD was significantly increased compared with 0 days $(p<0.01)$ after transfer from a favorable plant (cotton) to an unfavorable plant (tobacco). Our result is consistent with previous studies of Lymantria dispar SOD [40] and B. tabaci CuZnSOD [15]. We speculate from this result that MnSOD may assist the whitefly MEAM1 to adapt to new host plants, which might contribute to its global invasion.

Insecticides can induce oxidative stress, which is always a big challenge for insects. Zang et al. [41] compared the susceptibility of MEAM1 and indigenous ZHJ1 population of whitefly to imidacloprid and pyriproxyfen and found that ZHJ1 was more susceptible to insecticides than MEAM1. The low susceptibility to insecticides of MEAM1 may contribute to the whitefly's invasion and displacement of indigenous competitors. Interestingly, in our study, significant increase of MnSOD activity was found when MEAM1 fed on the cotton treated with imidacloprid. Similarly, Kemal [42] reported that low concentrations of malathion $(0.01-1 \mathrm{ppm})$ resulted in the significantly increased activity of SOD in Pimpla turionellae females compared with control. Altogether, we speculate that the MnSOD of 
whitefly MEAM1 might be involved in response to pesticides. In addition, the displacement of indigenous whitefly species by MEAM1 might be related to MnSOD activities, which can reduce the high level of superoxide radically induced by pesticides.

\section{Experimental Section}

\subsection{Whitefly Cultures and Plants}

Stock cultures of the invasive whitefly MEAM1, collected from Zhejiang, China, were established in the laboratory. Details of the methods for maintaining whitefly cultures were described by Jiu et al. [43]. The cultures were maintained on cotton plants (Gossypium hirsutum L. cv. Zhemian 1793) and regularly monitored for purity using random amplified polymorphic DNA polymerase chain reaction (RAPD-PCR) with the primer H16 (5'-TCTCAGCTGG-3') [44].

Cotton (cv. Zhemian 1793) and tobacco (Nicotiana tabacum cv. NC89), a suitable and an unsuitable host plant for the whitefly, respectively $[45,46]$, were used in this study. All plants were cultivated with a potting mix (a mixture of peat moss, vermiculite organic fertilizer and perlite in a 10:10:10:1 ratio by volume) in plastic pots in whitefly-proof glasshouses at $27 \pm 1{ }^{\circ} \mathrm{C}, 14 \mathrm{~h}$ light: $10 \mathrm{~h}$ darkness and $40 \%-60 \%$ relative humidity $(\mathrm{RH})$. Plants were used for experiments at the 5-6 true leaf stage.

\section{2. cDNA Synthesis and Cloning of the Bt-mMnSOD}

Total RNA was isolated using the SV total RNA isolation system (Promega, Madison, WI, USA), following the manufacturer's protocol. cDNA was synthesized using total RNA as a template with the SMART RACE cDNA Amplification Kit (Clontech, Palo Alto, CA, USA). Initially, partial Bt-mMnSOD cDNA sequence was obtained from the whitefly transcriptome deposited in NCBI (GenBank accession no. HP662057) [47]. The 5' and 3' ends of Bt-mMnSOD were amplified using the SMART RACE cDNA Amplification Kit with the 5' primer (5'-CTAAACAGCTGCTGAATATC-3') and 3' primer (5'-ATGTTTGCGTGCTCCAAAAA-3'), following the manufacturer's instructions. The amplified products were analyzed on $1.5 \%$ agarose gel, and the target band was purified by a PCR purification kit (Promega, Madison, WI, USA). Then the products were cloned into the pMD18-T vector (Takara, Dalian, China) for sequencing.

\subsection{Sequence Analysis}

The nucleotide and deduced amino acid sequences of the Bt-mMnSOD were analyzed by DNAMAN version 6 [48]. The sequence similarity of MnSODs from different species was analyzed and compared using the BLAST search program [49]. Theoretical isoelectric points and predicted molecular masses were calculated using Prot-Param tools [50]. The signal peptide was predicted by Signal P4.0 program [51]. The MITOPROT [52] and TargetP 1.1 [53] were used to predict whether the sequence contains any targeting sequence and where it locates. To compare MnSOD of whitefly and other species, related sequences were obtained from GenBank (Table 1) and aligned using Clustal W [54]. The phylogenetic relationship among the sequences was determined by reconstructing a protein phylogeny using MEGA4 [55]. The tree topology was evaluated by the bootstrapping method (1000 replications). 


\subsection{Overexpression and Purification of Bt-mMnSOD}

A pair of primers were designed to clone the open reading frame of the Bt-mMnSOD and then ligated to the pGEX-4T-3 expression vector. The sense primer was designed as 5'-GGATCCATGTTTGCGTGCTCCAAAAA-3' with a BamH1 site and the antisense primer 5'-GTCGACCTAAACAGCTGCTGAATATC-3' containing Sal1 site. The ligated product was then transformed into E. coli BL21 (DE3) pLysS cells for recombinant protein expression (Novagen, Madison, WI, USA).

Bacteria were grown in $800 \mathrm{~mL} \mathrm{LB}$ medium containing $0.1 \mathrm{~g} / \mathrm{mL}$ ampicillin at $37{ }^{\circ} \mathrm{C}$ until an optical density of 0.6 at $600 \mathrm{~nm}$ was reached. IPTG was added to a final concentration of $0.2 \mathrm{mM}$, and the culture was grown at $18{ }^{\circ} \mathrm{C}$ for $18 \mathrm{~h}$ for the induction of protein expression. Protein samples were analyzed by $12 \%$ SDS-PAGE, followed by staining with Coomassie brilliant blue. The Bt-mMnSOD protein with the GST tag was purified using the GST gene fusion system, as described [56]. The eluted protein content was measured by UV absorbance at $280 \mathrm{~nm}$, and the purity of the protein was evaluated by $12 \%$ SDS-PAGE.

\subsection{Thermostability of Purified Bt-mMnSOD}

The activity of the purified recombinant Bt-mMnSOD was determined using the CuZn/Mn SOD Assay Kit (Beyotime, Shanghai, China). The kit applied the 2-(4-iodophenyl)-3-(4-nitrophenyl)-5-(2, 4-disulfophenyl)-2 $H$-tetrazolium, monosodium salt (WST-1) method. The WST-1 could react with superoxide anion to generate water-soluble formazan dye. However, this reaction can be inhibited by SOD. Through colorimetric analysis of the WST-1 product, we can calculate the SOD enzyme activity. The absorbance was measured at $450 \mathrm{~nm}$ using spectrophotometer at room temperature. One unit of enzyme activity was defined as the amount of enzyme that results in 50\% inhibition of the xanthine oxidase coupled reaction under the assay condition. To determine the thermal stability of Bt-mMnSOD, reactions were carried out at $37,45,55,65,75$ and $85^{\circ} \mathrm{C}$ for 0 (control), 10, 20, 30, 40, 50 and $60 \mathrm{~min}$ and the relative activity of Bt-mMnSOD was measured, respectively. Each treatment was replicated for three times. A multiple comparisons (LSD) test was conducted to detect significant differences $(p=0.01)$ between the treatments using the DPS software [57].

\subsection{Bt-mMnSOD Gene Expression in Different Developmental Stages}

Quantitative real-time PCR (qPCR) was performed on the ABI PRISM 7500 Fast Real-Time PCR System (Applied Biosystems, USA). Total RNA was isolated from different developmental stages of B. tabaci, including egg \& nymph, $4^{\text {th }}$ instar and adult. RNA concentration was measured by NanoDrop 2000c (Thermo, Minneapolis, MN, USA). cDNA was synthesized using PrimeScript ${ }^{\circledR}$ RT reagent Kit (Takara, Dalian, China). The following primers, qPCR-F (5'-ACCACCGCTAA TCAAGATCC-3') and qPCR-R (5'-TGCAGGTAGTAGGCGTGTTC-3'), designed by the GenScript Real-time PCR Primer Design Software [58], were used for qPCR. As an endogenous control, the expression of $\beta$-actin was measured in parallel. 


\subsection{Effect of Thermal Stresses on the MnSOD Activity of the Whitefly}

Whiteflies were independently exposed to three different temperatures $\left(4,26,40{ }^{\circ} \mathrm{C}\right)$ for $0,30,60$ and $120 \mathrm{~min}$ in climatic chambers. The control and treated whiteflies were kept in liquid nitrogen immediately after treatment and were stored at $-80{ }^{\circ} \mathrm{C}$ until use. Fifty whiteflies were transferred into a tissue grinding tube with $200 \mu \mathrm{L}$ cell lysis buffer $(20 \mathrm{Mm}$ Tris, $\mathrm{pH} 7.5,150 \mathrm{mM} \mathrm{NaCl}, 1 \%$ Triton $\mathrm{X}-100)$. After grinding, the lysate was centrifuged at $4{ }^{\circ} \mathrm{C}$ and the supernatant was used for enzymatic assays. The activity of the MnSOD was determined using the CuZn/Mn-SOD Assay Kit (Beyotime, Shanghai, China). During the assay, CuZnSOD inhibitor A and B were added in the sample according to the manufacturer's protocol, so the result of the assay was only about MnSOD. The detailed procedures can be referred to in the protocol (Beyotime, Shanghai, China). Each treatment was replicated for three times. The statistical analysis is described in "Thermostability of purified Bt-mMnSOD".

\subsection{Effect of Host Shift on the MnSOD Activity of the Whitefly}

Whiteflies collected from cotton plants were transferred to tobacco plants and new cotton plants (control). After 0 (control), 1, 3 and 5 days, 50 whiteflies were collected in a $5 \mathrm{~mL}$ tube from tobacco and new cotton plants, respectively. The whiteflies were then treated with liquid nitrogen and stored at $-80{ }^{\circ} \mathrm{C}$. Each treatment was replicated for three times. The method used for preparing samples and the detection of enzyme activity is described in "Effect of thermal stresses on the MnSOD activity of the whitefly". The statistical analysis is described in "Thermostability of purified Bt-mMnSOD".

\subsection{Effect of Pesticide Treatment on MnSOD Activity of the Whitefly}

Three cotton plants were sprayed with $15 \mathrm{~mL}$ of imidacloprid (Dashifeng, Beijing, China). The concentration of active ingredients was $20 \mathrm{mg} / \mathrm{L}$. After $6 \mathrm{~h}$, two thousand adult whiteflies were transferred to these cotton plants. Thereafter, 50 whiteflies were collected in a $5 \mathrm{~mL}$ tube after 0 (control), 6, 12 and $24 \mathrm{~h}$, respectively. The whiteflies were then treated with liquid nitrogen and stored at $-80{ }^{\circ} \mathrm{C}$. Each treatment was replicated three times. The method used for preparing samples and the detection of enzyme activity is described in "Effect of thermal stresses on the MnSOD activity of the whitefly". The statistical analysis is described in "Thermostability of purified Bt-mMnSOD".

\section{Conclusions}

In this study, a mitochondrial manganese superoxide dismutase from the whitefly MEAM1 was cloned and characterized for the first time. Our results elucidated the important roles of MnSOD in the cellular stress responses and anti-oxidative processes of the whitefly MEAM1. The in vivo activity of Bt-mMnSOD under various conditions indicated the strong adaptive capacity of the invasive whitefly MEAM1 to extreme temperatures, host plants shift and insecticide treatment, which might contribute to its successful global invasion and continuous displacement of indigenous whitefly species. Future studies will be necessary to investigate functions of more antioxidant enzymes to gain a better understanding of the antioxidant mechanism in the whitefly species under various stresses. 


\section{Acknowledgments}

Financial support for this study was provided by the National Basic Research Program of China (Project 2009CB119203), the National Natural Science Foundation of China (Project 31071686) and the Qianjiang Talent Plan (2011R10012).

\section{Conflict of Interest}

The authors declare no conflict of interest.

\section{References}

1. Brown, J.K.; Frohlich, D.R.; Rosell, R.C. The sweetpotato or silverleaf whiteflies: Biotypes of Bemisia tabaci or a species complex? Annu. Rev. Entomol. 1995, 40, 511-534.

2. Perring, T.M. The Bemisia tabaci species complex. Crop Prot. 2001, 20, 725-737.

3. Boykin, L.M.; Shatters R.G., Jr.; Rosell, R.C.; McKenzie, C.L.; Bagnall, R.A.; de Barro, P.J.; Frohlich, D.R. Global relationships of Bemisia tabaci (Hemiptera: Aleyrodidae) revealed using Bayesian analysis of mitochondrial COI DNA sequences. Mol. Phylogenet. Evol. 2007, 44, 1306-1319.

4. De Barro, P.J.; Liu, S.S.; Boykin, L.M.; Dinsdale, A.B. Bemisia tabaci: A statement of species status. Annu. Rev. Entomol. 2011, 56, 1-19.

5. De Barro, P.J. Bemisia tabaci, a top 100 invader. J. Insect Sci. 2008, 8, 16.

6. Dalton, R. Whitefly infestations: The Christmas invasion. Nature 2006, 443, 898-900.

7. Liu, S.S.; de Barro, P.; Xu, J.; Luan, J.B.; Zang, L.S.; Ruan, Y.M.; Wan, F.H. Asymmetric mating interactions drive widespread invasion and displacement in a whitefly. Science 2007, 318, 1769-1772.

8. Dinsdale, A.; Cook, L.; Riginos, C.; Buckley, Y.M.; de Barro, P.J. Refined global analysis of Bemisia tabaci (Hemiptera: Sternorrhyncha: Aleyrodoidea: Aleyrodidae) mitochondrial cytochrome oxidase 1 to identify species level genetic boundaries. Ann. Entomol. Soc. Am. 2010, 103, 196-208.

9. Luo, C.; Yao, Y.; Wang, R.J.; Yan, F.M.; Hu, D.X.; Zhang, Z.L. The use of mitochondrial cytochrome oxidase I (mt COI) gene sequences for the identification of biotypes of Bemisia tabaci (Gennadius) in China. Acta Entomol. Sin. 2002, 45, 759-763.

10. Zhang, L.P.; Zhang, Y.J.; Zhang, W.J.; Wu, Q.J.; Xu, B.Y.; Chu, D. Analysis of genetic diversity among different geographical populations and determination of biotypes of Bemisia tabaci in China. J. Appl. Entomol. 2005, 129, 121-128.

11. Chu, D.; Zhang, Y.J.; Brown, J.K.; Cong, B.; Xu, B.Y.; Wu, Q.J.; Zhu, G.R. The introduction of the exotic $\mathrm{Q}$ biotype of Bemisia tabaci from the Mediterranean region into China on ornamental crops. Fla. Entomol. 2006, 89, 168-174.

12. Xu, J. Studies on the Invasion by Alien Bemisia tabaci in Zhejiang and Comparison of Biological Characteristics between Biotypes of the Whitefly. Ph.D. Thesis, Zhejiang University, Hangzhou, China, June 2009.

13. Oliveira, M.R.V.; Henneberry, T.J.; Anderson, P. History, current status and collaborative research projects for Bemisia tabaci. Crop Prot. 2001, 20, 709-723. 
14. Rosell, R.C.; Shah, A.; Khan, M.; Aghakasiri, N.; Shatters, R.G.; McKenzie, C.L. Superoxide dismutase activity in temperature stressed viruliferous whiteflies (Hemiptera: Aleyrodidae). In Preceeding of the XXIII International Congress of Entomology, Durban, South Africa, 6-12 July 2008.

15. Li, J.M.; Su, Y.L.; Gao, X.L.; He, J.; Liu, S.S.; Wang, X.W. Molecular characterization and oxidative stress response of an intracellular $\mathrm{Cu} / \mathrm{Zn}$ superoxide dismutase (CuZnSOD) of the whitefly, Bemisia tabaci. Arch. Insect Biochem. 2011, 77, 118-133.

16. McCord, J.M.; Fridovich, I. The utility of superoxide dismutase in studying free radical reactions. J. Biol. Chem. 1969, 244, 6056-6063.

17. Halliwell, B.; Gutteridge, J.M.C. Free Radicals in Biology and Medicine; Oxford University Press: Oxford, UK, 1999.

18. Mates, J.M.; Perez-Gomez, C.; de Castro, I.N. Antioxidant enzymes and human diseases. Clin. Biochem. 1999, 32, 595-603.

19. Brouwer, M.; Brouwer, T.H.; Grater, W.; Brown-Peterson, N. Replacement of a cytosolic copper/zinc superoxide dismutase by a novel cytosolic manganese superoxide dismutase in crustaceans that use copper (haemocyanin) for oxygen transport. Biochem. J. 2003, 374, 219-228.

20. Plantivaux, A.; Furla, P.; Zoccola, D.; Garello, G.; Forcioli, D.; Richier, S.; Merle, P.L.; Tambutté, É.; Tambutté, S.; Allemand, D. Molecular characterization of two CuZn-superoxide dismutases in a sea anemone. Free Radic. Biol. Med. 2004, 37, 1170-1181.

21. Bannister, J.V.; Bannister, W.H.; Rotilio, G. Aspects of the structure, function and application of superoxide dismutase. CRC Crit. Rev. Biochem. 1987, 22, 111-180.

22. Ken, C.F.; Lee, C.C.; Duan, K.J.; Lin, C.T. Unusual stability of manganese superoxide dismutase from a new species, Tatumella ptyseos ct: Its gene structure, expression and enzyme properties. Protein Expres. Purif. 2005, 40, 42-50.

23. Brouwer, M.; Brouwer, T.H.; Grater, W.; Enghild, J.J.; Thogersen, I.B. The paradigm that all oxygen-respiring eukaryotes have cytosolic $\mathrm{CuZn}$-superoxide dismutase and that $\mathrm{Mn}$-superoxide dismutase is localized to the mitochondria does not apply to a large group of marine arthropods. Biochemistry 1997, 36, 13381-13388.

24. Beyer, W.; Imlay, J.; Fridovich, I. Superoxide dismutases. Prog. Nucleic Acid Re. 1991, 40, 221-253.

25. Cheng, W.; Tung, Y.H.; Liu, C.H.; Chen, J.C. Molecular cloning and characterisation of cytosolic manganese superoxide dismutase (cytMn-SOD) from the giant freshwater prawn Macrobrachium rosenbergii. Fish Shellfish Immun. 2006, 20, 438-449.

26. Lin, Y.C.; Lee, F.F.; Wu, C.L.; Chen, J.C. Molecular cloning and characterization of a cytosolic manganese superoxide dismutase (cytMnSOD) and mitochondrial manganese superoxide dismutase (mtMnSOD) from the kuruma shrimp Marsupenaeus japonicus. Fish Shellfish Immun. 2010, 28, 143-150.

27. Sun, J.; Folk, D.; Bradley, T.J.; Tower, J. Induced overexpression of mitochondrial Mn-superoxide dismutase extends the life span of adult Drosophila melanogaster. Genetics 2002, $161,661-672$. 
28. Greenberger, J.S.; Epperly, M.W.; Gretton, J.; Jefferson, M.; Nie, S.; Bernarding, M.; Kagan, V.; Guo, H.L. Radioprotective gene therapy. Curr. Gene Ther. 2003, 3, 183-195.

29. Greenberger, J.S.; Epperly, M.W. Radioprotective antioxidant gene therapy: Potential mechanisms of action. Gene Ther. Mol. Biol. 2004, 8, 31-44.

30. Kim, Y.I.; Kim, H.J.; Kwon, Y.M.; Kang, Y.J.; Lee, I.H.; Jin, B.R.; Han, Y.S.; Cheon, H.M.; Ha, N.G.; Seo, S.J. Modulation of MnSOD protein in response to different experimental stimulation in Hyphantria cunea. Comp. Biochem. Phys. B 2010, 157, 343-350.

31. Wang, M.Q.; Su, X.R.; Li, Y.; Jun, Z.; Li, T.W. Cloning and expression of the Mn-SOD gene from Phascolosoma esculenta. Fish Shellfish Immun. 2010, 29, 759-764.

32. Boveris, A.; Chance, B. Mitochondrial generation of hydrogen-peroxide-general properties and effect of hyperbaric-oxygen. Biochem. J. 1973, 134, 707-716.

33. Cadenas, E.; Davies, K.J. Mitochondrial free radical generation, oxidative stress and aging. Free Radic. Bio. Med. 2000, 29, 222-230.

34. Harari, P.M.; Fuller, D.J.; Gerner, E.W. Heat-shock stimulates polyamine oxidation by 2 distinct mechanisms in mammalian-cell cultures. Int. J. Radiat. Oncol. 1989, 16, 451-457.

35. Rauen, U.; Polzar, B.; Stephan, H.; Mannherz, H.G.; de Groot, H. Cold-induced apoptosis in cultured hepatocytes and liver endothelial cells: Mediation by reactive oxygen species. FASEB J. 1999, 13, 155-168.

36. Banerjee Mustafi, S.; Chakraborty, P.K.; Dey, R.S.; Raha, S. Heat stress upregulates chaperone heat shock protein 70 and antioxidant manganese superoxide dismutase through reactive oxygen species (ROS), p38MAPK and Akt. Cell Stress Chaperon. 2009, 14, 579-589.

37. Dash, B.; Metz, R.; Huebner, H.J.; Porter, W.; Phillips, T.D. Molecular characterization of two superoxide dismutases from Hydra vulgaris. Gene 2007, 387, 93-108.

38. Futuyma, D.J.; Peterson, S.C. Genetic-variation in the use of resources by insects. Annu. Rev. Entomol. 1985, 30, 217-238.

39. Rossiter, M.C. Genetic and phenotypic variation in diet breadth in a generalist herbivore. Evol. Ecol. 1987, 1, 272-282.

40. Janković-Hladni, M.; Ivanović, J.; Spasić, M.; Blagojević, D.; Perić-Mataruga, V. Effect of the host plant on the antioxidative defence in the midgut of Lymantria dispar L. caterpillars of different population origins. J. Insect Physiol. 1997, 43, 101-106.

41. Zang, L.S.; Fu, R.X.; Liu, S.S.; Li, J.M.; Liu, Y.Q. Comparison of susceptibility to insecticides between the B biotype and a non B biotype of Bemisia tabaci in Zhejiang (In Chinese). Chin. Bull. Entomol. 2006, 43, 207-210.

42. Kemal, B. Malathion-induced oxidative stress in a parasitoid wasp: Effect on adult emergence, longevity, fecundity and oxidative and antioxidative response of Pimpla turionellae (Hymenoptera : Ichneumonidae). J. Econ. Entomol. 2006, 99, 1225-1234.

43. Jiu, M.; Zhou, X.P.; Tong, L.; Xu, J.; Yang, X.; Wan, F.H.; Liu, S.S. Vector-virus mutualism accelerates population increase of an invasive whitefly. PLoS One 2007, 2, e182.

44. DeBarro, P.J.; Driver, F. Use of RAPD PCR to distinguish the B biotype from other biotypes of Bemisia tabaci (Gennadius)(Hemiptera: Aleyrodidae). Aust. J. Entomol. 1997, 36, 149-152. 
45. Zang, L.S.; Chen, W.Q.; Liu, S.S. Comparison of performance on different host plants between the B biotype and a non-B biotype of Bemisia tabaci from Zhejiang, China. Entomol. Exp. Appl. 2006, 121, 221-227.

46. Xu, J.; Lin, K.K.; Liu, S.S. Performance on different host plants of an alien and an indigenous Bemisia tabaci from China. J. Appl. Entomol. 2011, 135, 771-779.

47. Wang, X.W.; Luan, J.B.; Li, J.M.; Su, Y.L.; Xia, J.; Liu, S.S. Transcriptome analysis and comparison reveal divergence between two invasive whitefly cryptic species. BMC Genomics 2011, 12, 458.

48. DNAMAN. Available online: http://www.lynnon.com (accessed on 10 June 2012).

49. BLAST Search Program. Available online: http://blast.ncbi.nlm.nih.gov/Blast.cgi (accessed on 1 March 2012).

50. Prot-Param Tools. Available online: http://kr.expasy.org/tools/protparam.html (accessed on 1 June 2012).

51. Signal P Program. Available online: http://www.cbs.dtu.dk/services/SignalP (accessed on 25 May 2012).

52. MITOPROT. Available online: http://ihg.gsf.de/ihg/mitoprot.html (accessed on 10 June 2012).

53. TargetP. Available online: http://www.cbs.dtu.dk/services/TargetP (accessed on 10 June 2012).

54. Clustal W. Available online: http://www.ebi.ac.uk/Tools/msa/clustalw2 (accessed on 1 May 2012).

55. MEGA. Available online: http://www.megasoftware.net (accessed on 1 April 2012).

56. Guan, K.L.; Dixon, J.E. Eukaryotic proteins expressed in Escherichia coli: An improved thrombin cleavage and purification procedure of fusion proteins with glutathione $S$-transferase. Anal. Biochem. 1991, 192, 262.

57. Tang, Q.Y.; Feng, M.G. DPS Data Processing System. Science Press: Beijing, China, 2007.

58. GenScript Real-time PCR Primer Design Software. Available online: https://www.genscript.com/ ssl-bin/app/primer (accessed on 27 June, 2012).

(C) 2013 by the authors; licensee MDPI, Basel, Switzerland. This article is an open access article distributed under the terms and conditions of the Creative Commons Attribution license (http://creativecommons.org/licenses/by/3.0/). 